\title{
A Possible Pairing Mechanism in Fe-Based High Temperature Superconductors
}

\author{
Ning Chen and Yang $\mathrm{Li}^{*}$
}

\author{
Department of Engineering Science and Materials, University of Puerto Rico at Mayaguez, Mayaguez PR 00681-9044, \\ USA
}

School of Material Science and Engineering, University of Science and Technology Beijing, Beijing 100083, China

Department of physics, University of Science and Technology Beijing, Beijing 100083, China

\begin{abstract}
The electronic structures of LaOFeAs superconductors with antiferromagnetic model were simulated by using the first-principle method. The calculated density of states (DOS) reveals that some antiferromagnetic energy peaks are splitted due to the hybridization of Fe-3 $d$ and As- $4 p$ on the FeAs layer. The calculated spin moment for LaOFeAs is 2.39 $\mu_{B} / \mathrm{Fe}$. The band structure and DOS around the Fermi level suggest this compound with a semimetal-like characteristic. Beside on the $\mathrm{Fe}$, a spin polarization occurs on the As site, which is distributed with both As- $4 p$ and As- $4 s$ orbitals. A unique electronic structure is found to be associated with the deep inner orbital coupling in the LaOFeAs compound, which provides an independent energy band with a high vibration frequency. The two bands with deep inner orbital coupling as well as the spin polarization of As- $4 s$ could be used to explain some behaviours of carrier pairing in the superconducting state. In addition, the simulation also was performed on a series of F-doped LaOFeAs with different fluorine composition. The results show with F-doping increasing the spin polarization of As decreasing. Superconductivity will be suppressed by the heavy F-doping concentration.
\end{abstract}

\section{INTRODUCTION}

Even now there is no sign that the pace of progress is slowing down. Despite the difficulty of working with these novel cuprate ceramic materials, applications to electrical cables, high field magnetics, motors, microwave filters, and electronics have been forthcoming. Indeed recent years have seen renewed interest in the field in following 1986 discovery of cuprate high temperature superconductivity [1]. The cuprate high temperature superconductors have transition temperatures as high as $160 \mathrm{~K}$ [2]. Furthermore an incredibly wide range of materials is now known to be superconducting. The field is no longer confined to the study of metallic elements and their alloys, but now includes the study of complex oxides, carbon-based materials such as fullerence $\mathrm{C}_{60}$, organic conductors, rare earth based compounds (heavy fermion materials), clathrate materials $\left(\mathrm{Ba}_{8} \mathrm{Si}_{46}\right)[3,4]$, and materials based on sulphur and boron $\left(\mathrm{MgB}_{2}\right)$ [5]. Recently, a new system of ferrugate LaOFeAs compounds showed evidence of superconductivity at high as $25 \mathrm{~K}[6,7]$, which provide rich fodders for research of high- $T_{C}$ superconductivity. The materials with large magnetic moments usually are not expected to be superconductors due to strong ferromagnetism which usually destroys electron pairing. Therefore, magnetism has long been thought to be incompatible with superconductivity. According to the Bardeen-CooperSchreiffer theory of superconductivity [8], electrons with opposite spins pair up and down are propelled through the superconductor by vibrations of the crystal lattice known as phonons. But magnetism changes the spin on the electrons and stops them pairing up. Even a very small number of

*Address correspondence to this author at the Department of Engineering Science and Materials, University of Puerto Rico at Mayaguez, Mayaguez PR 00681-9044, USA; E-mail: ylibp@hotmail.com and nchen@sina.com magnetic impurities can inhibit the formation of pairs. However, in cuprate superconductors, the evidences of $\mathrm{Cu}$ atom cannot be replaced and the antiferromagnetic background in the $\mathrm{Cu}-\mathrm{O}$ network demonstrate the high- $T_{C}$ superconductivity with some magnetic correlations [9]. The discovery of the ferrugate high temperature superconductor will provide an uncharted arena in which the magnetic correlations with high- $T_{C}$ superconductivity could be more directly visualized.

The Fe-based high temperature superconductors of quaternary oxy-pnictides have formula REOFeAs with a $\mathrm{P} 4 / \mathrm{nmm}$ tetragonal structure. The layered structure is composed of a series of stack $(R E O)_{2}$ and $(\mathrm{FeO})_{2}$. The structure and concentration of charge carriers can be modified by chemical doping. The rare earth $R E$ atom can be partially substituted by alkali-earth such as $\mathrm{Sr}$ to form a hole-doped superconductor with $T_{C}=25 \mathrm{~K}$ [8]. On the other hand, an electron-doped superconductor is prepared by substituting $\mathrm{O}$ with $\mathrm{F}$, its critical temperature is $25 \mathrm{~K}$ [7]. Both hole- and electron-doping can modulate the concentration of charge carriers and magnetic background in order to arise the superconducting temperatures to their maximum, $25 \mathrm{~K}$, which much higher than that in the systems of $\mathrm{LaONiP}\left(T_{C}=3 \mathrm{~K}\right)$ [10], $\mathrm{LaOFeP}\left(T_{C}=4 \mathrm{~K}\right)$ and $\mathrm{LaO}_{1-x} \mathrm{~F}_{x} \mathrm{FeP}\left(T_{C}=7 \mathrm{~K}\right)$ [11]. In addition, Zhao's group synthesized iron-based F-doped layered quaternary compound $\operatorname{Pr}\left[\mathrm{O}_{1-x} \mathrm{~F}_{x}\right] \mathrm{FeAs}$ with $T_{C}=52 \mathrm{~K}$ [12] which is the highest record in this kind of Fe-based superconducting system. Wen's group revealed the phase diagrams of electron-doped $\mathrm{La}\left(\mathrm{O}_{1-x} \mathrm{~F}_{x}\right) \mathrm{FeAs}$ and hole-doped $\left(\mathrm{La}_{1-x} \mathrm{Sr}_{x}\right) \mathrm{OFeAs}$ [8], which have some similarities with cuprate high temperature superconductors in $T_{C}$ changing with carrier concentration. Both work are very important not only in the hole-doping with same effect on superconductivity as electron-doping, but also in Fe-As network with similar action as $\mathrm{Cu}-\mathrm{O}$ planar layers in cuprate oxides. We have been 
deeming that it is very important for the magnetic correlation with superconductivity in cuprate superconductors. The antiferromagnetism on the $\mathrm{Cu}-\mathrm{O}$ is helpful for electron pairing at such high temperature but not mediated by phonons. The new discovery of Fe-contained superconductors demonstrates the importance of magnetic mediation again. It is clear that exploration of mechanism of superconductivity in Fe-contained compounds has the potential to reveal new superconductors with higher critical temperatures, and the ultimate quest for room temperature superconductors, in which the magnetic mediation could be critical.

In order to help clarify some important issues in the Fecontained superconductors, we present our simulation results by means of the first principle method for Fe-contained superconductors with different F-doping composition. In contrast to the electronic structures and magnetic moment distributions for different doped compounds, we find that the spin polarization occurs on As site, which plays an important role for superconductivity in Fe-contained compounds. In addition a unique electronic structure is found to be associated with the deep inner orbital coupling in LaOFeAs compound. In the view of the spin polarization of As- $4 s$ and the deep inner orbital coupling, a mentioned superconductivity mechanism could be used to explain the carrier pairing in the superconducting state.

\section{CALCULATION DETAILS}

In order to determine the electronic structure in the system of LaOFeAs, which is helpful for understanding the superconductivity in such Fe-contained compounds, and explain the effect of $\mathrm{F}$ doping on superconductivity, firstprinciples calculations for the periodic boundary systems were carried out using code developed by Payne et al. [13, 14]. This first-principle ab initio calculation package, using plane-wave basis sets and suited for periodic systems. The total energy is minimized with respect to occupied orbitals using a conjugate-gradients method. Either LDA (local density approximation) or GGA (generalized gradient approximation) were used to calculate the exchange and correlation energy of the electrons. Pseudopotentials are used to model the interaction of the valence electrons with the core of each atom. The total energy pseudopotential method we used is based on density functional theory (DFT) in describing the electron-electron interaction and on a pseudopotential description of the electron-core interaction. It gives the total electronic energy of a large system, as well as its band structure. Transferability and robustness of the assumed pseudopotentials of each element seem to be confirmed by success in reproducing the physical properties such as lattice parameters of many compounds. Therefore, it can be expected to give the relative stability of different crystal structures. We used the Payne's code in order to solve the pseudopotential Schrodinger equation self-consistently. Besides the F-doped La LaOFeAs compound mentioned above, F-doped $R E$ OFeAs ( $\mathrm{RE}=\mathrm{Pr}, \mathrm{Sm}$, and $\mathrm{Nd}$ ) compound were also calculated as a reference in order to check the suitability of the model parameters. We carried out first-principles calculations based on density functional theory (DFT) with generalized gradient approximation (GGA) of PBE-type [15] for the exchangecorrelation potential. Pseudopotentials are used to model the interaction of the valence electrons with the core of each atom, and the ultra-soft pseudopotential scheme is adopted.
The measured structural parameters [7] were selected as the input data for the model simulations. LaOFeAs has a layered tetragonal crystal structure with $\mathrm{P} 4 / \mathrm{nmm}$ symmetry. The basic lattice parameters are $a=4.035 \AA$ and $c=8.741$ $\AA$. The $(\mathrm{FeAs})^{-}$and $(\mathrm{LaO})^{+}$triple-layer-subgroups stack alternatively along the $c$-axis. In the $a-b$ plane, Fe atoms form a planar-square lattice with spacing of $2.853 \AA$. La and As layers are respectively positioned by two internal parameters, $z_{L a}=0.142$ and $z_{A s}=0.651$, which labelling the inter-layer distances of $\mathrm{La}-\mathrm{O}$ and $\mathrm{Fe}-\mathrm{As}$, respectively.

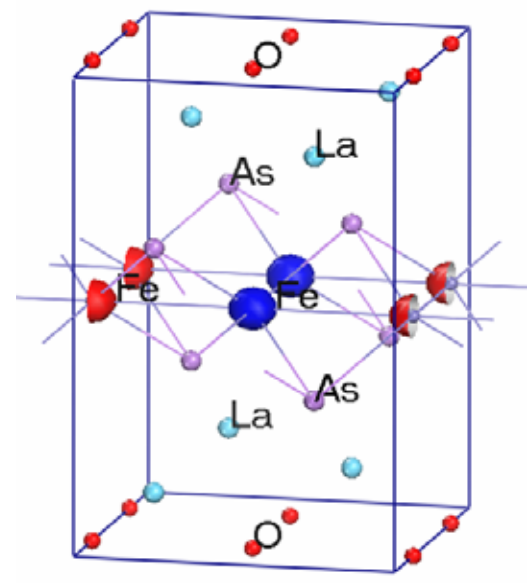

Fig. (1). Antiferromagnetic simulation model with superlattice structure including $4 \mathrm{Fe}$ atoms. The moment of Fe shows an antiferromagnetic arrangement. The red and blue ball represents Fe atom with spin up and down respectively.

\section{RESULTS}

In our simulation, the spin-polarization is considered for LaOFeAs. In order to discuss the antiferromagnetic ordering of $\mathrm{Fe}$ atoms in a larger space range, as shown in Fig. (1), a large superlattice model including $4 \mathrm{Fe}$ atoms is selected, in which the diagonal of basic lattice in the $a-b$ plane is set as the edge-length of superlattice

Two simulations are performed in order to consider the effect of different spin distributions on electronic structure. For the first one, the moments of Fe atom were set with antiferromagnetic constitution, which is compatible with neutron diffraction [16], while for the second one, spin moments were unfixed yet, which is adopted by other researchers in early time.

The band structure and density of states $N(E)$ for LaOFeAs model with antiferromagnetic constitution are shown in Fig. (2). The band structure exhibits semi-metallic characteristic. The density of state $N(E)$ shows a pseudogap with width about $0.3 \mathrm{eV}$ is located well on Fermi level $E_{\mathrm{F}}$. In contrast to the total energy calculated for two models, the antiferromagnetic model has a relatively smaller value by $0.362 \mathrm{eV}$ per Fe atom than the spin-unfixed model, which implied the antiferromagnetic constitution is more reasonable. A direct comparison to DOS $N(E)$ for two models can conclude that some peaks in the range of 0 to $-2 \mathrm{eV}$ are splitted (Fig. 2) origins from antiferromagnetic ordering of $3 d$ electron spin of $\mathrm{Fe}$ atoms. The pseudogap on $E_{\mathrm{F}}$ looks not explicit with a characteristic of semi-metal as shown in Fig. (2), which is compatible with the band structure. In addition, 

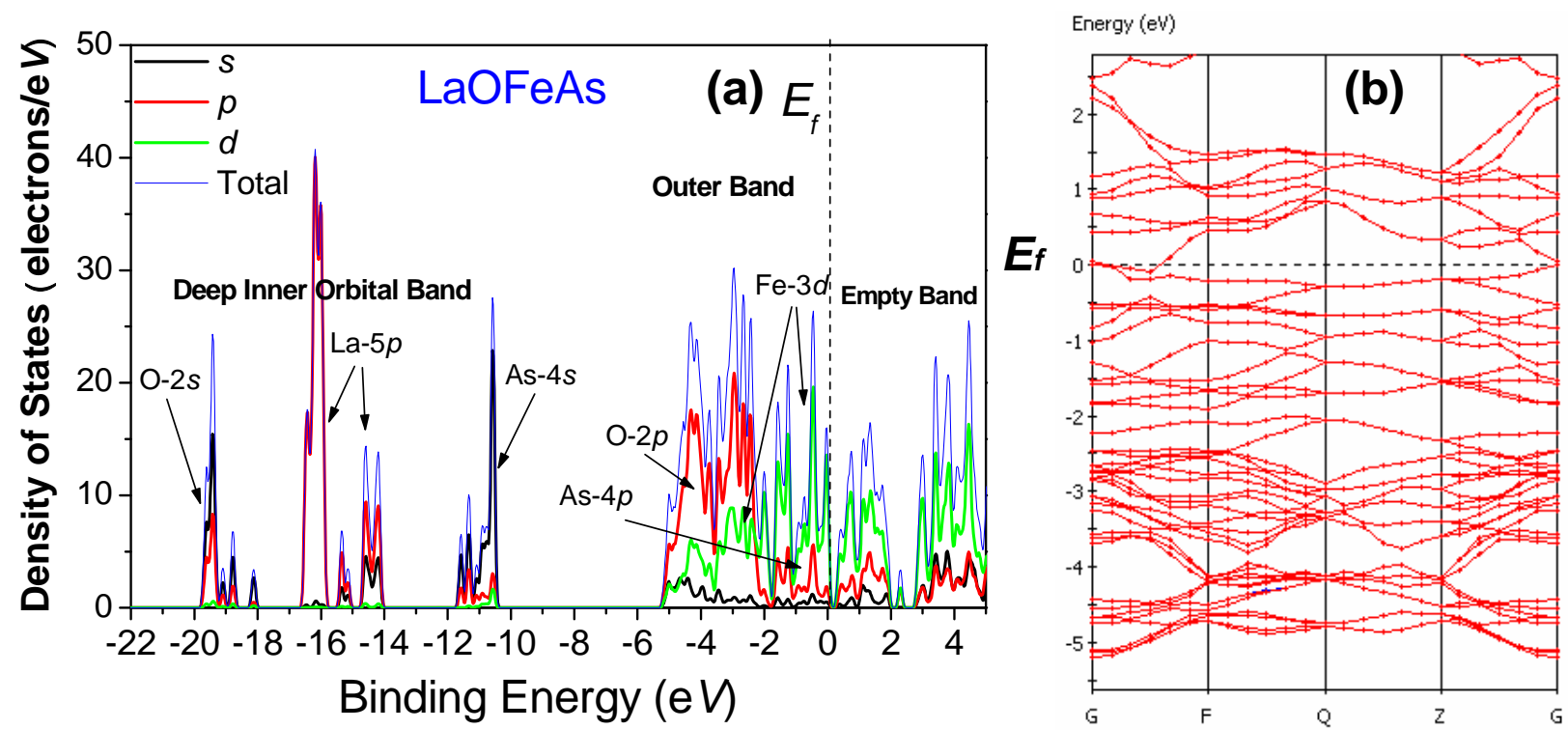

Fig. (2). (a) Density of states with total energy spectrum and (b) band structure for LaOFeAs, where $G=(0,0,0), F=(0,1 / 2,0), Q=(0,1 / 2,1 / 2)$, $Z=(0,0,1 / 2)$. Three deep inner bands are dominated by hybridization of O- $2 s$, La-5p and As- $4 s$ orbitals, respectively. The outer band in the vicinity of Fermi level arisen from the hybridization of $\mathrm{O}-2 p, \mathrm{Fe}-3 d$ and As- $4 p$ orbitals. The Fermi level is denoted by the dashed line.

the density of state $N(E)$ with a small value at the Fermi level implies a small concentration of carriers in the compound of $\mathrm{LaOFeAs}$, which is consistent with the experiments in Hall effect [17] and X-ray photoelectron spectroscopy (XPS) measurement [18].

The density of states with total energy spectrum for LaOFeAs is shown in Fig. (2). Combining with analysis of band structure and charge distribution, we identified three deep inner $N(E)$ bands arisen from the hybridization of O-2s, La-5 $p$ and As- $4 s$ orbitals, respectively. The outer band in the vicinity of Fermi level arisen from the hybridization of $\mathrm{O}-2 p$, Fe- $3 d$ and As- $4 p$ orbitals.

The $p$ - $d$ hybridization between $\mathrm{O}$ and Fe is very significant for this compound since it results in the pseudogaps opening near $E_{\mathrm{F}}$, as well as the anti-ferromagnetic ordering background induced by spin-polarization. For the DOS as shown in Fig. (2), the hybridization of As- $4 p$ and Fe-3d mainly is arisen from the electronic outer band in the range of 0 to $-2 \mathrm{eV}$. On the other hand, the band in the range of -2 to $-6 \mathrm{eV}$ has a large fraction from the contribution of $\mathrm{O}-2 p$ in
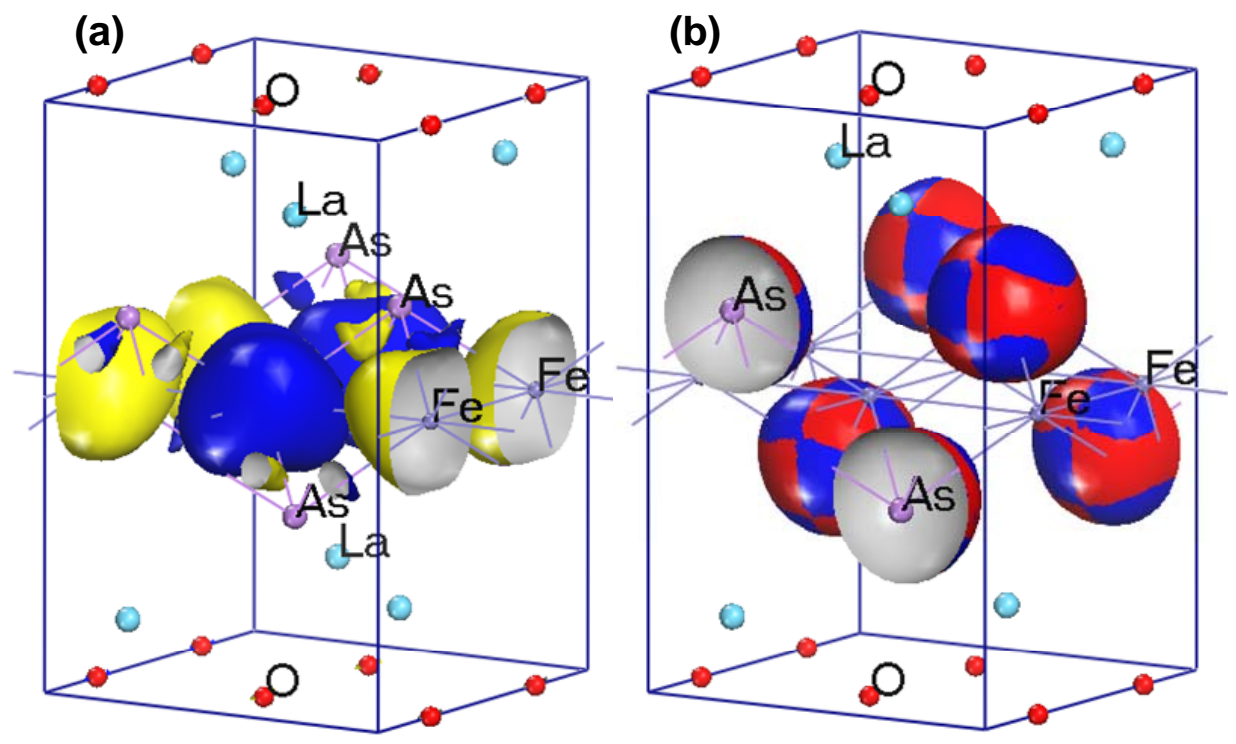

Fig. (3). (a) the contour map of total spin density distribution for LaOFeAs crystal. The blue and yellow represents spin up and down respectively, where isosurfaces corresponding to $0.030 \mathrm{e} / \AA^{3}$; (b) The contour map of electronic density for inner orbitals of As- $4 s$ with energy range from -12 to $-10 \mathrm{eV}$. The Blue and red represent $\alpha$ - and $\beta$-orbital, corresponding to spin-up and spin-down states, respectively. The isosurfaces corresponds to $0.050 \mathrm{e} / \AA^{3}$. 
La-O layer. It is particularly noted that this band is with a very similar characteristic like $\mathrm{O}-2 p$ in cuprate high $\mathrm{T}_{\mathrm{C}}$ superconductors in which the coupling of $\mathrm{O}-2 p$ and $\mathrm{Cu}-3 d$ brings on a super-exchange effect.

According to calculation of the anti-ferromagnetic model, the $p$ - $d$ hybridization between $\mathrm{O}$ and $\mathrm{Fe}$ produces a spin moment $2.39 \mu_{B} / \mathrm{Fe}$. Such spin moments of Fe-3d constitute an anti-ferromagnetic array in Fe-As layer, as shown in Fig. (3a). In addition, a sizable spin polarization of As- $4 p$ is also obtained as a result of hybridization of Fe-3d and As- $4 p$. However, the spin polarization of As- $4 p$ is not so strong like $\mathrm{Fe}-3 d$ due to only with the filled $p$-shell.

The spin density of the inner As- $4 s$ orbital with energy range from -12 to $-10 \mathrm{eV}$ is shown in Fig. (3b). The red and blue part represents $\alpha$ - and $\beta$-orbital respectively, which corresponding to the spin-up and -down state. It is clear that the red and blue parts jointly exist on As- $3 s$ orbital, which implies spin polarization of inner As-4s. Such polarization in inner As-3 $s$ orbital would be associated with large polarization of the outer As- $4 p$ orbital due to two orbitals in close vicinity in space even though they have a big interval in energy. Therefore, In Fe-As layer there occurs another set of magnetic lattice formed by the polarized inner As- $4 s$ orbital beside the outer Fe-As magnetic lattice.

In Fe-As network as a conduction layer, the As plays an important role for carriers moving. Since in the FeAs layer, the $\mathrm{Fe}$ ions are too remote from each other to have direct electron hoping and the As ion is needed to bridge the electron hoping between the Fe ions [19]. Between two antiferromagnetic moments of $\mathrm{Fe}$, the polarization direction of As certainly opposites to one of $\mathrm{Fe}$ moments, due to magnetic frustration, which will give rise to the magnetic skew scattering for carriers. The polarization of As no doubt will influence the transportation behaviour of carriers, which would be one of reasons for observed abnormity of Hall coefficient $R_{H}$ [20] It has been widely accepted that the temperature dependence of $R_{H}$ could be caused by a magnetic skew scattering mechanism, i.e. the scattering of conduction electrons from local moments is asymmetric due to spin-orbital coupling. In fact, the magnetic skew scattering has been observed in various materials with the presence of magnetic moments. As the compound contains Fe element, the calculation shows the presence of Fe local moments, then the skew scattering mechanism might work there [21].

The spin density distributions of $\mathrm{LaO}_{1-x} \mathrm{~F}_{x} \mathrm{FeAs}$ superlattice model (model including $8 \mathrm{Fe}$ atoms) with different $\mathrm{F}$ doping composition are shown in Fig. (4). For $x=0,0.125$, 0.25 and 0.5 model, there are $0,1,2$ and 4 fluorine atoms substitute for oxygen in superlattice respectively. In Fig. (4), along the array direction of $\mathrm{Fe}$ ion with the same spin direction, we can see that there always is an As ion with opposite spin direction between two Fe ions. An antiferromagnetic arrangement of As-Fe is formed. The spin polarization in-
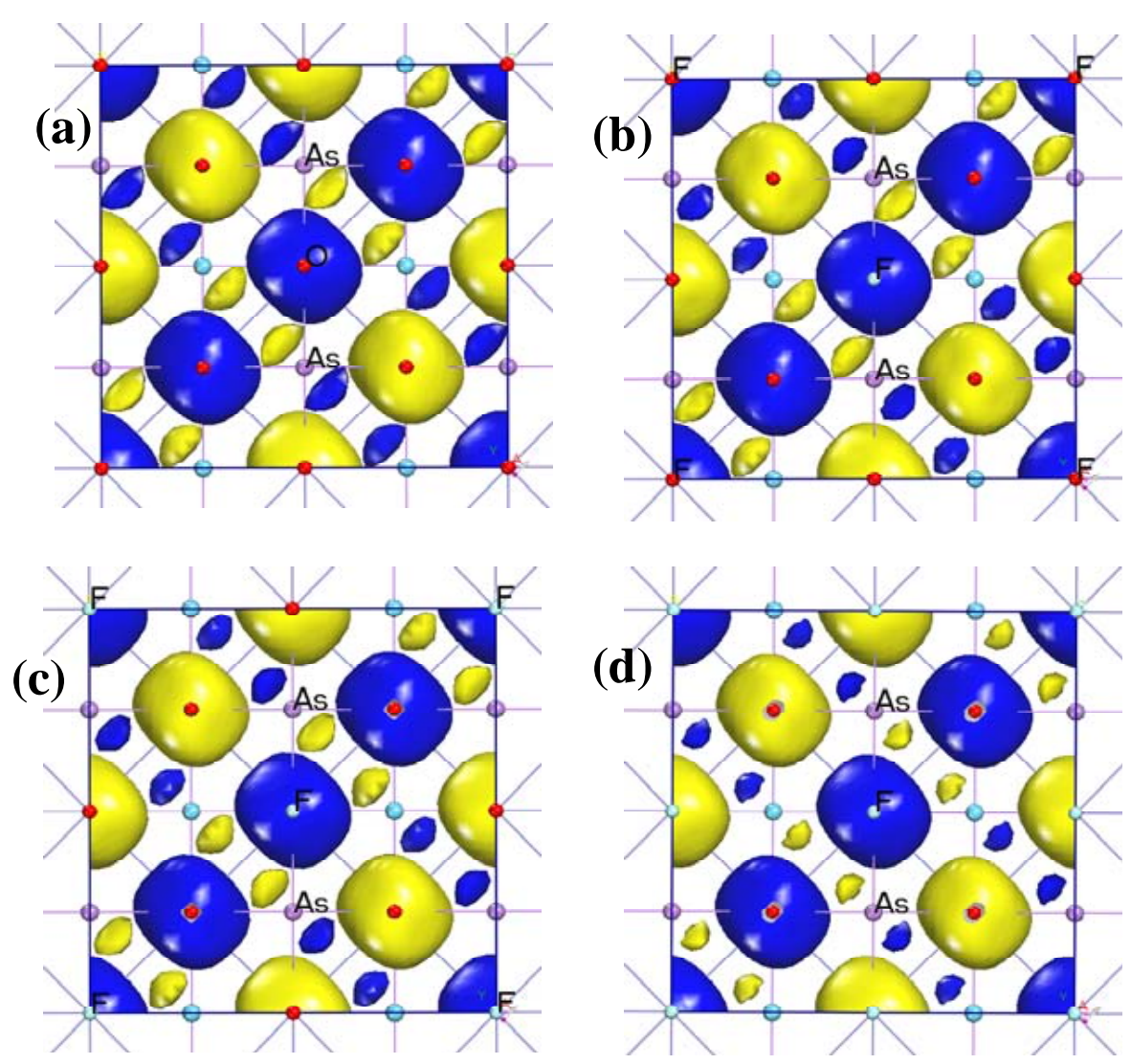

Fig. (4). Comparison of spin density distribution of $\mathrm{LaO}_{1-x} \mathrm{~F}_{x} \mathrm{FeAs}$ with different composition $x$, (a) $x=0$, (b) $x=0.125$, (c) $x=0.25$ and (d) $x=0.5$ model. The polarization on the As site is induced by hybridizing between As- $4 p$ and Fe- $3 d$, which like a bridge connecting with two magnetic Fe ions. With the increase of doped concentration, the polarization of As decreases implying the bridge is gradually broken. The yellow and blue balls are Fe ions with spin-up and spin-down respectively, in which isosurfaces corresponding to $0.030 \mathrm{e} / \AA^{3}$. 
duced by the hybridization of As- $4 p$ and Fe-3d like a bridge connecting two magnetic Fe ions. In contrast of the spin density distribution for $\mathrm{La}\left(\mathrm{O}_{1-x} \mathrm{~F}_{x}\right) \mathrm{FeAs}$ compounds with different fluorine concentrations, it shows the doping concentration does not influence the antiferromagnetic ordering, but Fdoping suppresses the spin polarization of As. With the increase of F-doping concentration, the polarization of As tends toward smaller, which implies for heavy F substitution this bridge is gradually broken. Apparently, F-doping in $\mathrm{LaOFeAs}$ will increase the concentration of carrier which is of benefit to superconductivity, but heavy substitution destroys the spin polarization of As, which as a bridge of $\mathrm{Fe}$ ions. Considering superconductivity is related with the concentration of carrier as well as the polarization of As, it is not difficult to understand why for the moderate F-doping such as $x=0.125$ is the optimal concentration with the higher critical temperature $T_{C}$ [7]. Under this doping composition, both the concentration of carriers and magnetic ordering can keep in a moderate range for superconductivity.

\section{DISCUSSIONS}

From the total density of states in the range of -20 to -10 $\mathrm{e} V$ as shown in Fig. (5a), we noticed the deep inner-orbital coupling band which origins the orbital coupling between $\mathrm{O}$ $2 s$ and La-5p (and As- $4 s$ and La-5p). Here we specially use "deep inner orbital coupling" in order to distinguish it with other "inner orbital coupling" with energy above $-6 \mathrm{eV}$ such as $\mathrm{As}-4 p$ and $\mathrm{Fe}-3 d$.
For the deep inner orbital coupling, as shown in Fig. (5), we notice that electrons occupy O- $2 s$ and La-5p states at same time. Both DOS and electronic charge density distribution indicate that intensive coupling between O- $2 s$ and La-5p orbital, like as a covalent bonding. For the coupling of As- $4 s$ and La-5p, we also can obtain the same conclusion. From the DOS, we can see that the exchanging population is quite large. In addition, the electronic charge distribution in real space shows the inner-orbital coupling can extend in entire space as in a super-long molecular. Due to such long-range coupling, provided the electronic vibration is excited in the coupling band, these excited quasi-particles could propagate in longrange without energy loss. We speculate this mechanism to occur in the LaOFeAs and cuprate high- $T_{\mathrm{C}}$ superconductors. In addition, since the lattice position is fixed by valence bonds of outer orbitals, the inner-orbital only associates with electronic vibration but without nucleus shift. According to the energy of inner orbital coupling, this vibration frequency would be much larger than that of phonon; the estimated frequency is about 10 times bigger than phonon frequency. Our results show that a unique two-band electronic structure plays important roles in high- $T_{C}$ superconductivity. The two-band is composed of the valance band and a deep inner coupling band which was simply overlooked before.

Provided the inner-orbital coupling is suitable for vibration with high frequency, the sub-magnetic lattice such as As-4s mentioned above, could supply for a stable excitation at high temperature. We consider that the local moment on the As site
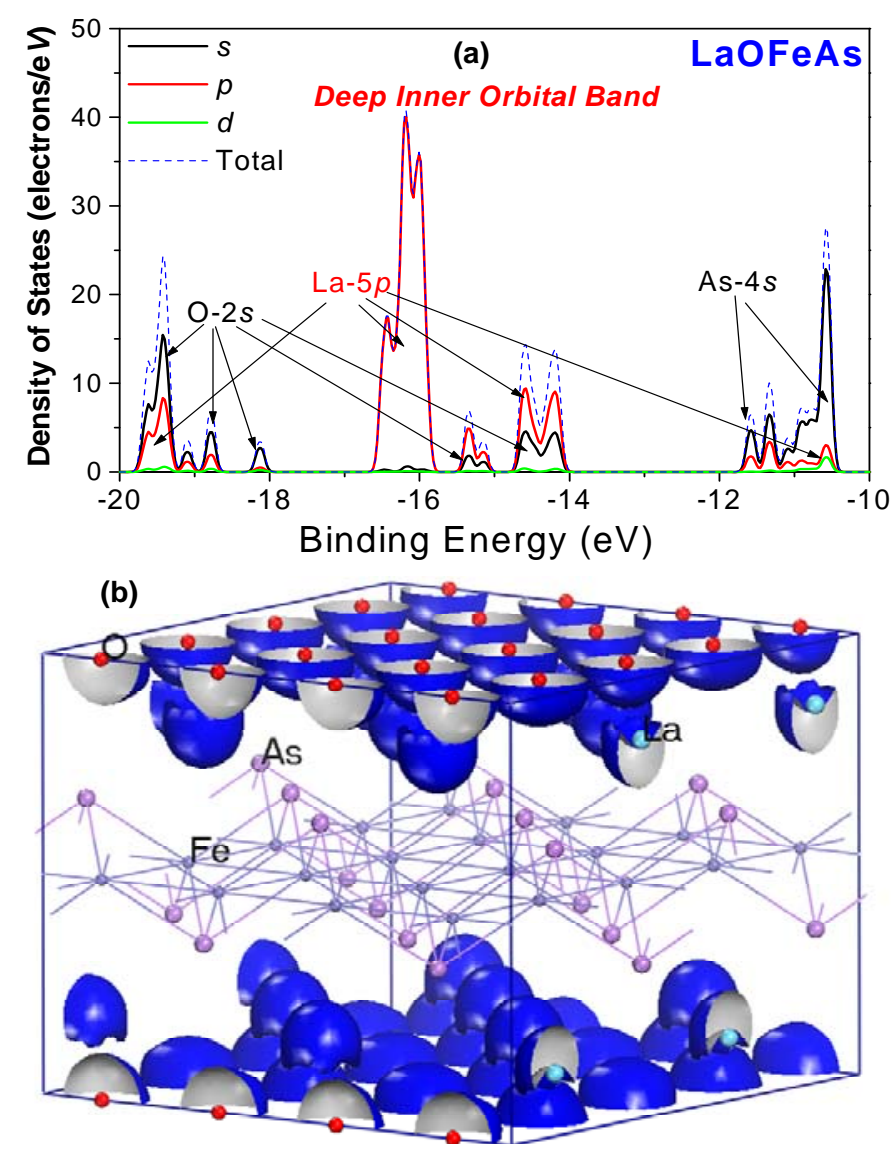

Fig. (5). (a) the DOS peaks of deep inner orbitals in the range of -20 to $-10 \mathrm{e} V$ for LaOFeAs model; (b) the contour map of electronic density distribution for one inner orbital O- $2 s$ corresponding to the DOS peak at $-19.5 \mathrm{eV}$, isosurfaces correspond to $0.030 \mathrm{e} / \AA^{3}$. Both two figures indicate the intensive inner-orbital coupling between $\mathrm{O}-2 s$ and La- $5 p$. 
in the sub-magnetic lattice, can act as two magnetic fences with opposite magnetic direction due to its antiferromagnetic characteristic. One of them could slow down electrons with spin up, the other one could influence the electrons with spin down. So neither up-spin nor down-spin could go through these fences easily, which can explain the anomaly effect of resistivity for normal state. But if two electrons with opposite spin direction combine into one non-magnetic electron pair ( $\operatorname{spin}=0$, charge $=2 e$ ), the electron-pair can pass through the magnetic fence smoothly with a lower dynamic energy, even though their potential energy be elevated as a results of electronic compelling with each other. Therefore, in view of electron structure, there occurs a deep inner-band corresponding to a high-frequency, which produces a sub-antiferromagnetic background. Such magnetic interaction with carriers can influence their dynamic behaviour, i.e. Hall coefficient occurs to anomaly in normal state, and electron pairing realizes by means of "antiferromagnetic fence" in superconducting state.

\section{CONCLUSIONS}

We reported the electronic structures of $\mathrm{LaOFeAs}$ superconductors with antiferromagnetic model. The Fermi level of calculated DOS reveals this compound as a semi-metal. The calculated spin distribution suggests that some density peaks are splitted by the super-exchanging of $\mathrm{Fe}-3 d$ and As- $4 p$ on the FeAs layer. The calculated spin moment for LaOFeAs is $2.39 \mu_{B} / \mathrm{Fe}$. A spin polarization occurs on the As site, which is distributed with both As- $4 p$ and As- $4 s$ orbitals. A very special electronic structure is found to be associated with the deep inner orbital coupling from La-5 $p, \mathrm{O}-2 s$ and As- $4 s$ in $\mathrm{LaOFeAs}$ compound, which may provide an independent energy band with a high vibration frequency. This deep innerorbital coupling as well as the spin polarization of As- $4 s$ could be used to explain the carrier pairing in the superconducting state. In addition, the simulation also was performed in the Fdoping LaOFeAs. The results show with F-doping increasing the spin polarization of As decreasing. Superconductivity will be suppressed for the heavy F-doping concentration.

\section{ACKNOWLEDGEMENTS}

This work was supported by the National Science Foundation under Grant No. DMR-0821284 and 0351449 (PREM program), National Natural Science Foundation of China (Grant No.50372005) and National Natural Science Foundation of Beijing (Grant No. 1072007).

\section{REFERENCES}

[1] Bednorz JG, Muller KA. Possible high Tc superconductivity in the barium-lanthanum-copper-oxygen system. Z Phys 1986; B 64: 18993.

[2] Gao L, Xue YY, Chen F, et al. Superconductivity up to $164 \mathrm{~K}$ in $\mathrm{HgBa}_{2} \mathrm{Ca}_{m-1} \mathrm{Cu}_{m} \mathrm{O}_{2 m+2+\delta}(m=1,2$, and 3) under quasihydrostatic pressures. Phys Rev 1994; B50: 4260-3.

[3] Kawaji H, Horie HO, Yamanaka S, Ishikawa M. Superconductivity in the silicon clathrate compound $(\mathrm{Na}, \mathrm{Ba})_{\mathrm{x}} \cdot \mathrm{Si}_{46}$. Phys Rev Lett 1995; 74: 1427-9.

[4] Li Y, Zhang RH, Liu Y, et al. Superconductivity in galliumsubstituted $\mathrm{Ba}_{8} \mathrm{Si}_{46}$ clathrates. Phys Rev 2007; B75: 054513-8.

[5] Nagamatsu J, Nakagawa N, Muranaka T, Zenitani Y, Akimitsu Y Strongly linked current flow on polycrystalline forms of the new superconductor $\mathrm{MgB}_{2}$. Nature 2001; 410: 63-4.

[6] Kamihara Y, Watanabe T, Hirano M, Hosono H. Iron-Based Layered Superconductor $\mathrm{La}\left[\mathrm{O}_{1-x} \mathrm{~F}_{x}\right] \mathrm{FeAs}(x=0.05-0.12)$ with $T_{\mathrm{c}}=26$ K. J Am Chem Soc 2008; 130: 3296-7.

[7] Wen HH, Mu G, Fang L, Yang H, Zhu XY. Superconductivity at $25 \mathrm{~K}$ in hole-doped $\left(\mathrm{La}_{1-\mathrm{x}} \mathrm{Sr}_{\mathrm{x}}\right) \mathrm{OFeAs}$. Eur Phys Lett 2008; 82: 17009 .

[8] Bardeen J, Cooper LN, Schrieffer JR. Theory of superconductivity. Phys Rev 1957; 108: 1175-1204.

[9] Chen N, Zhang LG, Li Y. Spin distributions on $\mathrm{CaCuO}_{2}$ cuprate high-Tc superconductor. Physica 2008; B403: 1142-3.

[10] Watanabe T, Yanagi H, Kamiya T, et al. Nickel-based oxyphosphide superconductor with a layered crystal structure, LaNiOP. Inorg Chem 2007; 46: 7719-21

[11] Kamihara Y, Hiramatsu H, Hirano M, et al. Iron-based layered superconductor: LaOFeP. J Am Chem Soc 2006; 128: 10012-3.

[12] Ren ZA, Yang J, Lu W, et al. Superconductivity at $52 \mathrm{~K}$ in ironbased F-doped layered quaternary compound $\mathrm{PrO}_{1-\mathrm{x}} \mathrm{F}_{\mathrm{x}} \mathrm{FeAs}$. Mater Res Innov 2008; 12: 106-3.

[13] Payne MC, Teter MP, Allan DC, Arias TA, Joannopoulos JD. Iterative minimization techniques for $a b$ initio total-energy calculations: molecular dynamics and conjugate gradients. Rev Modern Phys 1992; 64: 1045-97.

[14] Segall MD, Lindan PJD, Probert MJ, et al. First principles methods using CASTEP. J Phys: Cond Matt 2002; B14: 2117-23.

[15] Perdew JP, Burke K, Ernzerhof M. Generalized gradient approximation made simple. Phys Rev Lett 1996; 77: 3865-8.

[16] de la Cruz C, Huang Q, Lynn JW, et al. Magnetic Order versus superconductivity in the Iron-based layered $\mathrm{La}\left(\mathrm{O} 1{ }_{-} \mathrm{F}_{x}\right) \mathrm{FeAs}$ systems. Nature 2008; 453: 899-902.

[17] Chen GF, Li Z, Li G, et al. Superconducting properties of Fe-based layered superconductor $\mathrm{LaO}_{0.9} \mathrm{~F}_{0.1}-\delta \mathrm{FeAs}$. Phys Rev Lett 2008; 101 : 057007.

[18] Ou HW, Zhao JF, Zhang Y, et al. Angle integrated photoemission study of $\mathrm{SmO}_{0.85} \mathrm{~F}_{0.15} \mathrm{FeAs}$. Chin Phys Lett 2008; 25: 2225 -27.

[19] Li T. Orbital Degeneracy and the Microscopic Model of the FeAs Plane in the Iron-Based Superconductors. J Phys: Cond Matt 2008; 20: 425203.

[20] Zhu X, Yang H, Fang L, Mu G, Wen H-H. Upper critical field, Hall effect and magnetoresistance in the iron-based layered superconductor $\mathrm{LaFeAsO}_{0.9} \mathrm{~F}_{0.1-\delta}$. Supercond Sci Technol 2008; 21: 105001-5.

[21] Ong NP. The Hall Effect and its relation to the other transport phenomena in the normal state of high temperature super conductors. In: Ginsberg DM, Ed. Physical properties of high temperature superconductors II, World Scienfific Publishing Co 1990; 2: 459. 